\title{
Analysis of the Left Atrial Appendage by Three-Dimensional Transesophageal Echocardiography
}

Hideki Nakajima, RDCS, Yoshihiro Seo, MD ${ }^{\mathrm{a},{ }^{*}}$, Tomoko Ishizu, MD ${ }^{\mathrm{a}}$, Masayoshi Yamamoto, MD ${ }^{\mathrm{a}}$, Tomoko Machino, MD ${ }^{\mathrm{a}}$, Yoshie Harimura, MD , Ryo Kawamura, $\mathrm{MD}^{\mathrm{a}}$, Yukio Sekiguchi, $\mathrm{MD}^{\mathrm{a}}$, Hiroshi Tada, $\mathrm{MD}^{\mathrm{a}}$, Kazutaka Aonuma, $\mathrm{MD}^{\mathrm{a}}$

Department of Clinical Laboratory, Tsukuba University Hospital and ${ }^{\mathrm{a} C a r d i o v a s c u l a r}$ Division, Institute of Clinical Medicine, Graduate School of Comprehensive Human Sciences, University of Tsukuba, Ibaraki, Japan

Running head: 3D-TEE analysis of left atrial appendage morphology

Sources of support: No

*Corresponding author:

Yoshihiro Seo, MD

Cardiovascular Division, Institute of Clinical Medicine,

Graduate School of Comprehensive Human Sciences

University of Tsukuba

1-1-1 Tennodai, Tsukuba 305-8575, JAPAN

Phone: +81-298-53-3142

Fax: +81-298-53-3143

E-mail: yo-seo@md.tsukuba.ac.jp 


\section{Abstract:}

This study was designed to determine the ability and reliability of three-dimensional (3D) transesophageal echocardiography (TEE) to assess geometry and size of left atrial appendage (LAA). 3D-TEE may allow more accurate assessment of, and provide additional information on, LAA morphology as compared to two-dimensional TEE. Validation studies for LAA morphology data derived from 3D-TEE were performed using 10 isolated porcine LAA specimens. Of enrolled 107 patients, 55 patients were sinus rhythm (NSR), in whom 3D-TEE images were obtained from full-volume mode imaging, and in 52 patients with atrial fibrillation (AF), zoom mode imaging was used. We assessed LAA orifice area, depth, volume, and number of LAA lobes on reconstructed 3D imaging. LA volume was calculated using 2D echocardiographic imaging. In experimental studies, excellent correlations $(\mathrm{R}>0.90$, and $\mathrm{P}<0.001)$ between the measurements by full-volume or zoom mode imaging and reference data were observed in all parameters. The LAA orifice long diameter was $29.7 \pm 7.4 \mathrm{~mm}$, short diameter was $20.6 \pm 5.9 \mathrm{~mm}$, orifice area was $5.1 \pm 2.5 \mathrm{~cm}^{2}$, LAA depth was $38.5 \pm 8.2$ $\mathrm{mm}$, and volume was $9.2 \pm 5.6 \mathrm{ml}$. The most frequent number of LAA lobes was 2 ( $n=52)$, followed by $3(n=28), 1(n=18)$, and $4(n=3)$. LA volume weakly correlated with LAA orifice long diameter $(\mathrm{R}=0.33, \mathrm{P}=0.001)$, short diameter $(\mathrm{R}=0.40, \mathrm{P}<0.001)$, area $(\mathrm{R}=0.39, \mathrm{P}<0.001)$, LAA depth $(\mathrm{R}=0.21, \mathrm{P}=0.03)$, and volume $(\mathrm{R}=0.36, \mathrm{P}=0.001)$. 3D-TEE is a reliable modality to evaluate LAA geometry, and provides detailed information to quantify varied LAA characteristics.

Key Words: left atrial appendage, 3-dimensional transesophageal echocardiography, left atrium, morphology 
As an intracardiac source of emboli, the left atrial appendage (LAA) is a critical site of thrombus formation. ${ }^{1}$ Two-dimensional (2D) transesophageal echocardiography (TEE) has been widely used to characterize LAA structure and evaluate LAA function. ${ }^{2}$ Pathologic specimens of the LAA display its complex and extremely variable geometry. ${ }^{3,4}$ Now, three-dimensional (3D)-TEE can provide unique views using a pyramidal ultrasound beam ${ }^{5}$ and slice the image in any plane to enable visualization of any or all cardiac structures from multiple vantage points. ${ }^{6}$ The initial study with 3D-TEE showed the potential to assess LAA morphology more accurately as compared to 2D-TEE studies. ${ }^{7}$ However, detailed LAA morphology has not been fully examined with 3D-TEE. Therefore, this study was designed to determine the ability and reliability of 3D-TEE to assess the geometry and size of the LAA.

\section{Methods}

Three-dimensional datasets of LAA morphology in 3D-TEE examinations were validated using 10 isolated LAA specimens obtained from edible porcine hearts. The LAA reference volume was determined by measuring the volume of saline required to fill each LAA specimen. Reference long and short diameters of the LAA orifice were measured as the maximum and minimum lengths of the orifice. LAA depth was measured as the maximum length from the orifice to the leading edge of the lobes, and LAA orifice area also was measured with software (Scion Image, Scion Corporation, Frederick, MD). All data were measured by an observer (YS) who was blinded to the 3D-TEE data. Each LAA was suspended and placed into a water tank filled with de-aerated saline. The distance from TEE probe placed in the tank to porcine LAA specimens was set to approximately $5 \mathrm{~cm}$. 3D images were obtained for each LAA via full-volume mode and zoom mode examinations, and the long and short diameter of the LAA orifice, depth, orifice area, and LAA volume were each measured by an observer 
(HN) who was blinded to the reference data. Details of the method are summarized below in the method section of human study.

Consecutive 107 patients ultimately found not to have LAA thrombus were studied during clinically indicated TEE examinations to assess the presence of a LAA thrombus at Tsukuba University Hospital. LAA thrombi were assessed using a real-time 3D-TEE mode that can visualize two orthogonal images simultaneously (X-plane mode). Informed consent was obtained at the time of consent for the clinical TEE procedure.

Standard 2D-TTE examinations were performed with an iE33 ultrasound system and an S5-2 probe (Philips Medical Systems, Andover MA). The left ventricular (LV) end-diastolic and -systolic diameters were measured on an M-mode image derived from a LV short-axis view at the chordal level. LV end-diastolic and -systolic volume, and LV ejection fraction were measured using a modified-Simpson's method. ${ }^{8}$ End-systolic and -diastolic LA volumes were measured using a modified-Simpson's method in the apical 4-chamber and 2-chamber views. ${ }^{9}$

3D-TEE was performed using an iE33 ultrasound system with an S7-2 probe (Philips Medical Systems). LAA emptying flow velocity was measured in the basal short-axis view from the transverse scan ( $45^{\circ}$ views). The LAA emptying flow velocity was measured as the average of 3 consecutive cardiac cycles in patients with normal sinus rhythm (NSR) and 5 consecutive cardiac cycles in patients with atrial fibrillation (AF). Full-volume mode examinations were performed from $45^{\circ}$ views during apnea at end expiration. To obtain these data sets, 6 sectors were scanned with gating to the electrocardiographic $\mathrm{R}$ wave and were automatically integrated into a wide-angle $(76 \times$ 69 degrees) pyramidal data image covering the entire LAA. The frame rate of each image was set at approximately 20 to 30 frames/s. In patients with AF during the examination, zoom mode, which magnified the pyramidal scan by 1 cardiac beat, was 
used. The range of pyramidal scan was carefully regulated to include the entire LAA. The frame rate of each image was set at approximately 10 frames/s.

Quantification of LAA morphology was performed with QLAB GI-3DQ software (Philips). First, multi reconstruction planes of the LAA were obtained from 3D data sets (pyramidal images) including the LAA at the end-systole. In the long-axis view (Figure 1), the LAA orifice was determined by two lines; one was connected between the vestibule of the mitral valve annulus near the left coronary artery and the lateral ridge of the left superior pulmonary vein (LSPV), and another was between a point near the aortic valve annulus and the lateral ridge of the LSPV. ${ }^{10,11}$ We measured the LAA orifice long and short diameters, and orifice area at LV end-systole and -diastole (Figure 1). The LAA depth of the orifice to a lobe tip, and the angle of the first bend from the center of the LAA orifice on the center line to measure the LAA depth were measured at LV end-systole. LAA volume was measured at LV end-systole on a reconstructed image as follow: the inner-border of the LAA was manually traced within the distal area of the orifice, and the transverse images of the longest axis of the LAA trace area were automatically sliced at 10 levels from the orifice to the most distal site. On each sliced transverse image, the inner border was manually traced and reconstructed into a 3D image. On a reconstructed image, we measured LAA volume, and the number of LAA lobes was assessed based on the definition by Veinot et al. ${ }^{5}$ : 1) LAA lobe was a visible out-pouching from the main tubular body of the LAA, usually demarcated by an external crease; 2) it was internally capable of admitting a 2-mm probe (i.e., it was not simply a tag of external adipose tissue); 3) it was occasionally but not necessarily associated with a change in direction of the main tubular body of the LAA; 4) it could lie in a different anatomic plane than the main tubular body; and 5) by definition, the LAA must have at least one lobe (Figure2). 
Twenty studies were selected for assessment of intra- and inter-observer reproducibility of LAA volume measurements by 3D-TEE. Because different methods of acquiring 3D datasets were used between patients with NSR and those with AF, we studied 10 patients with NSR and 10 with AF. In all measurements, two observers independently obtained MPR images to assess LAA morphology from pyramidal 3D datasets. To test intra-observer variability, a single observer $(\mathrm{HN})$ analyzed the data twice on occasions separated by an interval of 1 month. To test inter-observer variability, a second observer analyzed the data without knowledge of the first observer's measurements. Reproducibilities were assessed as the mean percent error (absolute difference divided by the mean of the two observations).

Results are expressed as number (\%) or mean \pm SD. Comparisons of continuous variables between groups were performed with Student's t-test. Correlations between two variables were evaluated by linear regression analysis. Correlations between LA volume and the number of LAA lobes were evaluated by Pearson's rank correlation coefficient. A P value $<0.05$ was considered to indicate statistical significance. All calculations were performed with SPSS for Windows (SPSS Inc., Chicago, IL).

\section{Results}

Comparisons between the measurements at full-volume mode and zoom mode by 3D-TEE and reference data are shown in Figure 3. Excellent correlations were observed between echocardiographic data and reference data for each 3D-TEE mode.

All 107 patients underwent TEE without complications. Clinical characteristics and 2D-TTE data are summarized in Table 1. In 3D-TEE examinations, LAA morphology evaluations could be assessed in 101 patients (94\%). The remaining 6 patients, who all had AF rhythm, were excluded because of poor image quality for analysis. The clinical and echocardiographic data for the 6 excluded patients are summarized in Table2. 
Compared to included patients with AF, the heart rate (HR) was faster in the excluded patients, and all patients with HR $\geq 90$ bpm (Case Nos. 1, 3, 5, and 6) were excluded.

Comparisons of LA and LAA data between patients with NSR and AF are summarized in Table 3. In patients with AF, LA and LAA volume and LAA orifice measurements were larger than those in patients with NSR. Both in patients with NSR and in those with AF, LA volume and LAA orifice measurements at end-diastole were significantly decreased compared to those at end-systole. The number of LAA lobes ranged between 1 to 4 lobes, and a representative case of each is shown in Figure 4 . In patients with NSR, 2 lobes were observed most frequently $(n=30)$, followed by 1 lobe in 14 patients, 3 lobes in 10 patients, and 4 lobes in 1 patient. In patients with AF as well, patients with 2 lobes were observed most frequently $(n=22)$, followed by 3 lobes in 18 patients, 1 lobe in 4 patients, and 4 lobes in 2 patients.

Correlation between LA volume and parameters of LAA morphology are shown in Figure 5. LA volume was weakly correlated with LAA orifice area, LAA depth, LAA emptying flow velocity, and LAA volume, but not with the number of LAA lobes. In addition, these correlations were not significant in separate analysis among patients with NSR alone or those with AF alone.

In 10 patients with NSR, intraobserver variabilities in measurement were $4.8 \pm 3.2 \%$ for LAA long diameter, $4.5 \pm 3.0 \%$ for short diameter, $6.3 \pm 4.0 \%$ for LAA orifice area, $6.4 \pm 4.2 \%$ for LAA depth, and $6.8 \pm 4.6 \%$ for LAA volume, and interobserver variabilities were $6.7 \pm 5.7 \%, 6.4 \pm 5.4 \%, 7.2 \pm 6.0 \%, 8.2 \pm 6.8 \%$, and $7.3 \pm 6.5 \%$, respectively. In 10 patients with $\mathrm{AF}$, intraobserver variabilities were $6.3 \pm 6.0 \%$ for LAA long diameter, $4.8 \pm 2.8 \%$ for short diameter, $6.5 \pm 5.5 \%$ for LAA orifice area, 7.2 $\pm 4.0 \%$ for LAA depth, and $7.1 \pm 5.3 \%$ for LAA volume, and interobserver variabilities were $6.8 \pm 6.2 \%, 6.3 \pm 3.9 \%, 8.3 \pm 6.0 \%, 9.1 \pm 6.4 \%$, and $9.0 \pm 6.9 \%$, respectively. The 
number of LAA lobes corresponded among the 2 observers in all patients.

\section{Discussion}

In the present study, 3D-TEE provided feasible visualization of the LAA in the majority patients. In particular, adequate 3D full-volume images could be obtained in all patients with NSR. In contrast, adequate images could not be obtained in 6 patients (5.6\%) with AF. Similarly, in the first report on LAA visualization by 3D-TEE, adequate 3D LAA images could not be obtained in $5 \%$ patients. $^{7}$ Because of the irregular rhythm, the full-volume image derived from 6 cardiac beats was not available in patients with $\mathrm{AF}$, in whom we used zoom mode imaging. In comparison with included patients with AF, excluded patients had increased HR. The ability of zoom mode to assess highly mobile structures such as vegetations is limited. ${ }^{12}$ Therefore, the lower frame rate may affect image quality in patients with AF and increased HR. ${ }^{7}$ Despite such limitations, adequate images could be obtained in most patients with AF, and intra- and inter-observer variabilities in patients with AF were clinically acceptable. These results suggest LAA 3D-imaging to be available for the assessment of LAA geometry in the majority of patients referred for TEE.

The present study is the first to show detailed morphological variations of the LAA with 3D-TEE as previously reported in autopsy studies and studies with computed

tomography (CT). ${ }^{10,13-16}$ Our experimental studies, which revealed the accuracy of LAA measurements with 3D-TEE imaging, strongly support the reliability of our clinical results, although our results were not validated with other diagnostic imaging modalities such as cardiac multidetector CT.

With recent development of LAA occlusion systems, it has become clinically important to accurately assess LAA orifice size measurement to perform the correct sizing and placement of occlusion devices. ${ }^{7,10,15-17}$ 2D-TEE has been used to determine 
the device size. ${ }^{17}$ However, 3D-TEE estimated LAA orifice size more accurately. ${ }^{7}$ Although even 3D-TEE images should be used cautiously because of changes in LAA orifice area between proximal and distal portions of the LAA, the reconstructed whole images of the inner surface of the LAA used in our study may provide additional information on LAA anatomy so as to avoid suboptimal sizing of LAA occlusion devices. ${ }^{16}$ In addition the present study revealed LAA orifice morphology was changed throughout the cardiac cycle. The findings also may contribute to determine the optimal size of LAA occlusion device. Based on these abilities of the 3D-TEE, we consider that 3D-TEE provides more useful information for LAA occlusion device than 2D-TEE. In contrast, it is difficult to anatomically determine the LAA orifice, because of lack of LAA orifice ridge except for ridge-like fold of the atrial wall that separates the os from the left upper pulmonary vein. ${ }^{14}$ Therefore, our method for measurements of LAA orifice may differ from those of previous studies.

Furthermore, the present study showed the variability of LAA lobes. In an autopsy study, distribution of the number of LAA lobes was 2 (54\%), 3 (23\%), 1 (20\%), and 4 lobes (3\%), which agreed well with our results. ${ }^{3}$ In identifying the number of lobes, an important issue was to differentiate them from pectinate muscle, and we carefully determined LAA lobe based on pathological study. ${ }^{4}$ The clinical implications of LAA lobe variability have not been fully understood. In the future, 3D-TEE imaging studies may contribute to recognition of the implications of LAA lobe variability.

The present study showed modest relations between LAA morphology and LA volume.

Heist et al. ${ }^{18}$ also reported with magnetic resonance imaging that LAA depth and volume did not correlate with LA volume.

The LAA volume is normally about $10 \%$ of that of the entire LA volume, and the 
LAA is considered to play a part in LA function. ${ }^{19}$ However in some patients, the LAA is markedly dilated despite slight dilation of the LA main chamber. ${ }^{20}$ LAA is more compliant than the LA main chamber and has a role as reservoir for LA volume and pressure overload. ${ }^{21-23}$ Differences in compliance, which are attributed to quite different behaviors including distinct histologic structures between LA and LAA, may be a cause of the disturbances between LA volume and LAA morphology. ${ }^{24}$ In addition, the present study showed that patients with AF had larger LAA than those in patients with NSR as in previous studies. ${ }^{15,16}$ However, increases of LAA volume in patients with AF were significant but little compared to those of LA volume. The results suggest that AF has less effect on LAA remodeling.

Integration of a 3D full-volume image requires 6 cardiac cycles. Therefore, beat-by-beat motion artifacts, so-called stitching noise, may affect the 3D-images. Since a main cause of stitching noise is motion variation with respiration, we obtained all images during apnea at end expiration. In addition, since magnitudes of LAA motion are less than those of the LV and mitral valve through a cardiac cycle, the full-volume LAA images were less affected by the stitching noise. We did not assess the affect of LAA motion magnitudes by LAA fibrillation to obtain the LAA 3D images. The rapid motion during LAA fibrillation may affect accuracy of the LAA geometric measurements.

The experimental study may not be a comparable gold standard for the In-Vivo imaging due to the better penetration of the ultrasound beam that can be achieved in a water tank as opposed to the internal environment in a human subject.

\section{Acknowledgments}

The authors greatly appreciate the assistance of the echocardiographic laboratory staff Ms Hiroko Kobayashi, Mrs. Fumiko Sakamaki, Ms Satomi Yanaka, and Mrs. Noriko Iida. 
1. Odell JA, Blackshear JL, Davies E, Byrne WJ, Kollmorgen CF, Edwards WD, Orszulak TA. Thoracoscopic obliteration of the left atrial appendage: potential for stroke reduction. Ann Thorac Surg 1996;61:565-569.

2. Suetsugu M, Matsuzaki M, Toma Y, Anno Y, Maeda T, Okada K, Konishi M, Ono S, Tanaka N, Hiro J. Detection of mural thrombi and analysis of blood flow velocities in the left atrial appendage using transesophageal two-dimensional echocardiography and pulsed Doppler flowmetry [Japanese]. J Cardiol 1988;18:385-394.

3. Veinot JP, Harrity PJ, Gentile F, Khandheria BK, Bailey KR, Eickholt JT, Seward JB, Tajik AJ, Edwards WD. Anatomy of the normal left atrial appendage: A quantitative study of age-related changes in 500 autopsy hearts: implications for echocardiographic examination. Circulation 1997;96:3112-3115.

4. Kerut EK. Anatomy of the left atrial appendage. Echocardiography 2008;25:669-73.

5. Sugeng L, Shernan SK, Salgo IS, Weinert L, Shook D, Raman J, Jeevanandam V, Dupont F, Settlemier S, Savord B, Fox J, Mor-Avi V, Lang RM. Live 3-dimensional transesophageal echocardiography initial experience using the fully-sampled matrix array probe. J Am Coll Cardiol 2008;52:446-449.

6. Agoston I, Xie T, Tiller FL, Rahmen AM, Ahmad M. Assessment of left atrial appendage by live three-dimensional echocardiography: early experience and comparison with transesophageal echocardiography. Echocardiography 2006;23:127-132.

7. Shah SJ, Bardo DM, Sugeng L, Weinert L, Lodato JA, Knight BP, Lopez JJ, Lang RM. Real-time three-dimensional transesophageal echocardiography of the left atrial appendage: initial experience in the clinical setting. J Am Soc Echocardiogr 2008;21:1362-1368.

8. Nosir YF, Vletter WB, Boersma E, Frowijn R, Ten Cate FJ, Fioretti PM, Roelandt JR. 
The apical long-axis rather than the two-chamber view should be used in combination with the four-chamber view for accurate assessment of left ventricular volumes and function. Eur Heart J 1997;11:1175-1185.

9. Lang RM, Bierig M, Devereux RB, Flachskampf FA, Foster E, Pellikka PA, Picard MH, Roman MJ, Seward J, Shanewise JS, Solomon SD, Spencer KT, Sutton MS, Stewart WJ. Recommendations for chamber quantification: a report from the American Society of Echocardiography’s Guidelines and Standards Committee and the Chamber Quantification Writing Group, developed in conjunction with the European Association of Echocardiography, a branch of the European Society of Cardiology. J Am Soc Echocardiogr 2005;18:1440-1463.

10. Budge LP, Shaffer KM, Moorman JR, Lake DE, Ferguson JD, Mangrum JM. Analysis of in vivo left atrial appendage morphology in patients with atrial fibrillation: a direct comparison of transesophageal echocardiography, planar cardiac CT, and segmented three-dimensional cardiac CT. J Interv Card Electrophysiol 2008;23:87-93.

11. Cabrera JA, Ho SY, Climent V, Sánchez-Quintana D. The architecture of the left lateral atrial wall: a particular anatomic region with implications for ablation of atrial fibrillation. Eur Heart J 2008;29:356-362.

12. Perk G, Lang RM, Garcia-Fernandez MA, Lodato J, Sugeng L, Lopez J, Knight BP, Messika-Zeitoun D, Shah S, Slater J, Brochet E, Varkey M, Hijazi Z, Marino N, Ruiz C, Kronzon I. Use of real time three-dimensional transesophageal echocardiography in intracardiac catheter based interventions. J Am Soc Echocardiogr 2009;22:865-880. 13. Ernst G, Stollberger C, Abzieher F, Veit-Dirscherl W, Bonner E, Bibus B, Schneider B, Slany J. Morphology of the left atrial appendage. Anat Rec 1995;242:553-561. 14. Su P, McCarthy KP, Ho SY. Occluding the left atrial appendage: anatomical considerations. Heart 2008;94:1166-1170. 
15. Wongcharoen W, Tsao HM, Wu MH, Tai CT, Chang SL, Lin YJ, Lo LW, Chen YJ, Sheu MH, Chang CY, Chen SA. Morphologic characteristics of the left atrial appendage, roof, and septum: implications for the ablation of atrial fibrillation. J Cardiovasc Electrophysiol 2006;17:951-956.

16. Lacomis JM, Goitein O, Deible C, Moran PL, Mamone G, Madan S, Schwartzman D. Dynamic multidimensional imaging of the human left atrial appendage. Europace 2007;9:1134-1140.

17. Holmes DR, Reddy VY, Turi ZG, Doshi SK, Sievert H, Buchbinder M, Mullin CM, Sick P. Percutaneous closure of the left atrial appendage versus warfarin therapy for prevention of stroke in patients with atrial fibrillation: a randomized non-inferiority trial. Lancet 2009;374:534-542.

18. Heist E, Refaat M, Danik S, Holmvang G, Ruskin JN, Mansour M. Analysis of the left atrial appendage by magnetic resonance angiography in patients with atrial fibrillation. Heart Rhythm 2006;3:1313-1318.

19. Yamamoto N. Experimental study of combined left atrium resection for lung cancer [Japanese]. Nippon Kyobu Geka Gakkai Zasshi 1986;34:958-965.

20. Tabata T, Oki T, Yamada H, Iuchi A, Ito S, Hori T, Kitagawa T, Kato I, Kitahata H, Oshita S. Role of left atrial appendage in left atrial reservoir function as evaluated by left atrial appendage clamping during cardiac surgery. Am J Cardiol 1998;81:327-332. 21. Hondo T, Okamoto M, Yamane T, Kawagoe T, Karakawa S, Yamagata T, Matsuura H, Kajiyama G. The role of the left atrial appendage. A volume loading study in open-chest dogs. Jpn Heart J 1995;36:225-234.

22. Davis CA 3rd, Rembert JC, Greenfield JC Jr. Compliance of left atrium with and without left atrium appendage. Am J Physiol 1990;259:1006-1008.

23. Hoit BD, Shao Y, Tsai LM, Patel R, Gabel M, Walsh RA. Altered left atrial 
compliance after atrial appendectomy. Influence on left atrial and ventricular filling. Circ Res 1993;72:167-175.

24. Seto TB, Buchholz WA, Douglas PS, Manning WJ. When the body and appendage of the left atrium disagree: "Focal” atrial fibrillation implications for atrial thrombus formation and risk of thromboembolism. J Am Soc Echocardiogr 1999;12:1097-1100. 


\section{Figure legends}

Figure 1. Left atrial appendage views and measurements. Panel A represents a left atrial appendage (LAA) long-axis view at the level including the mitral valve annulus, left coronary artery (black arrow), and lateral ridge of the left superior pulmonary vein (white arrowhead). Panel B represents a LAA long-axis view at the level including the mitral valve annulus, aortic valve annulus (white arrows) and lateral ridge of the left superior pulmonary vein (white arrowhead). The dashed line with double-headed white arrows in Panels A and B corresponding to the line to determine the orifice of the LAA as shown in Panel C. In Panel B, the double-headed white arrow shows LAA depth and the angle of the first bend from the LAA orifice. LAA orifice area and long and short diameters are shown in Panel C.

Figure 2. Comparison of image quality between full-volume mode and zoom mode imaging. Panels A and B represent long-axis and short-axis images of the left atrial appendage (LAA) on full-volume mode, and Panels $\mathrm{C}$ and $\mathrm{D}$ the long-axis and short-axis images on zoom mode imaging, respectively. Each image clearly provides an image of the orifice of a LAA lobe (dashed circle) separate from other orifices. The green line shows a coronal section line for ultrasonic beam direction (Panels A and C), and the red line shows a sagittal section (Panels B and D).

Figure 3. Correlations of left atrial appendage (LAA) measurements with experimental study. Panel A presents correlations of LAA measurements from full-volume mode imaging with those of the experimental study. Panel B presents correlations of LAA measurements by zoom mode with those of the experimental study. Panel C presents correlations between measurements from full-volume mode and those from zoom mode. 
Figure 4. Representative reconstructed three-dimensional images of the left atrial appendage (LAA). Examples of LAAs with 1 lobe (Panel A), 2 lobes (Panel B), 3 lobes (Panel C), and 4 lobes (Panel D). The green line indicates the coronal section line for ultrasonic beam direction (Panels B, C and D), the red line indicates a sagittal section (Panel D), and the blue line indicates a horizontal section (Panels C and D).

Figure 5. Relation between left atrial (LA) volume and parameters of left atrial appendage (LAA) morphology. Regression lines and data means of the relations among all subjects are shown. Open circles = patients with normal sinus rhythm; closed circles $=$ patients with atrial fibrillation. 